\title{
Determining Parameters of an RLC Circuit Response to a Single Pulse
}

\author{
A. OzGA* \\ AGH - University of Science and Technology, Department of Mechanics and Vibroacoustics \\ al. A. Mickiewicza 30, 30-059 Krakow, Poland
}

\begin{abstract}
Experimental determining of distributions of pulses forcing a linear system, where pulse amplitudes and occurrence instants are random values, is burdened with errors resulting from uncertainty of the measurement and the differences between the model and the physical phenomenon. The objective of this work is an attempt to minimize these errors through application of an approximation algorithm that allows to determine parameters of response of the system to a single pulse forcing. The conclusions issuing from the investigations indicate that the parameters of the vibrating system should be selected so that the impact of the local deformations that occur while the system is being forced on the parameters of the system response should be as small as possible.
\end{abstract}

DOI: 10.12693 /APhysPolA.123.1034

PACS: $45.05 .+\mathrm{x}, 45.05 .+\mathrm{e}$

\section{Introduction}

Determining, in a finite time, the distributions of values of stochastic pulses forcing a one-dimensional physical system the state of which is described by means of a single parameter $x$ varying with time $t$ according to the equation of motion

$$
\frac{\mathrm{d}^{2} x}{\mathrm{~d} t^{2}}+2 b \frac{\mathrm{d} x}{\mathrm{~d} t}+a^{2} x=f(t)
$$

where $a$ and $b$ are constants, and the forcing function $f(t)$ is a series of pulses amplitudes and occurrence instants are random values, is a complex task. There exists a mathematical model [1-3] that allows for computing of distributions from a single motion waveform. Both in simulations and in experiments it is worth noticing that, independently of the intensity of the pulses or the parameters $a$ and $b$ of the vibrating system, in the initial moments of motion following each "hit" of the pulsed forcing, significantly changing the values of the stochastic moments, exerts its influence on the error that will burden the determination of the desired distribution. Experiments are additionally burdened with errors resulting from differences between the mathematical model and response of the physical system as well as the uncertainty of the measurement which constitutes another difficulty as regards the precision of calculations required in the model.

Although it is known that any model is merely a certain simplification of an actual physical phenomenon and therefore its usefulness is limited, it is hard to explain why different stochastic instants of occurrence and therefore different distributions of pulses are obtained in spite of application of the same distributions of hits in both theoretical and experimental investigations [4]. The

\footnotetext{
*e-mail: aozga@agh.edu.pl
}

higher is the intensity of these hits, the greater are these differences, and therefore it is incorrect to adopt the same substitute parameters $a$ and $b$ in an analysis of a single system forced by pulses of different intensities.

The subject of this paper is the approximation algorithm that allows for determining of the parameters of the system response to forcing with a single pulse. It is necessary to develop approximation techniques anew in a way making possible to define the physical phenomena that take place in an actual physical system while it vibrates being forced by a single pulse. This will subsequently lead to determining how the responses of the system change the moment when the intensity of pulses is altered.

\section{Mathematical model of the impulse value}

The solution of Eq.(1) for any $f(t)$ and $a>b$ (subcritical damping) assumes the form $[1,2]$

$$
\begin{gathered}
x(t)=\exp (-b t)\left(x_{0} \cos (c t)+\frac{\dot{x}_{0}+b x_{0}}{c} \sin (c t)\right) \\
+\frac{1}{c} \int_{0}^{t} f(\tau) \exp (-b(t-\tau)) \sin (c(t-\tau)) \mathrm{d} \tau,
\end{gathered}
$$

where $c=\sqrt{a^{2}+b^{2}}$, and

$$
x_{0}=x(0), \dot{x}_{0}=\frac{\mathrm{d} x}{\mathrm{~d} t}(0)
$$

represent initial conditions.

Let a single pulse forcing be considered in the following. In the literature of the subject [5], the physically interpretable quantity characterizing a very (infinitely in the theory) short pulse is the impulse $I$ of the forcing function $f(t)$ defined as

$$
I=\int_{0}^{\varepsilon} f(t) \mathrm{d} t
$$

where $\varepsilon$ is a time interval representing duration of the pulse. 
To date, authors of scientific studies dealing with vibrations of linear systems forced by a single pulse have been univocal. There exist two different models $[5,6]$ of a pulsed forcing $f(t)$, and it is the model representing the pulse with the help of Dirac delta function [5] that seems to be more intuitive. Although the two models differ as regards the methods of solving the integral in the equation (2), in the final version they arrive at the same conclusion that forcing with a pulse is equivalent to evoking free vibrations through a sudden increase of the initial velocity of the system, i.e. rate of change of parameter $x$ determining its state, without changing the parameter itself. This means that $x=x_{0}$ also immediately after a "hit" of the pulsed forcing. Under such assumption, motion of the system after occurrence of the pulsed forcing can be described by means of function

$$
x(t)=\eta \exp (-b t) \sin (c t),
$$

where $\eta=I / c$. The above formula is used, among others, in the studies that allow for determining the distribution of striking pulses $[1-3,7,8]$ from the waveform representing motion of a given vibrating system. Assumptions and proof the relevant theory can be found in $[1,2,7]$. This article will discuss the difficulties connected with realization and approximation of the response of the system to a pulsed forcing.

\section{Vibrations of an RLC circuit by a single pulse}

For practical reasons, mechanical representations of the system described by Eq. (1) are discussed most frequently, but in order to register actual response of a system of that kind to a single pulse, its electric counterpart is considered in the following. The study was carried out on a system consisting of inductivity $L=5 \mathrm{mH}$, capacity $C=5 \mathrm{nF}$, and a voltage source. All the elements were connected in series, and the resistance $R$ came from the physical components making up the circuit. The measuring unit comprised a measuring computer and a measuring card NI USB-6251 by National Instruments. The application was built in the Labview 9 environment. The single pulse was realized with the help of single samples of the shortest executable duration of $2 \times 10^{-6} \mathrm{~s}$, related to the sampling rate of the card.

The greatest difficulty occurs as early as the level of executing the experiment - the execution of the force that achieves high values and operates for such a short period of time that the system responds to its action with a change of the initial values only is impossible. Every measuring card has its characteristics of increase to a given value and decrease from this value to zero. In view of this, the process of forcing vibrations in a real system by a pulse may be divided into three phases (see Fig. 1 ). The first phase is characterized by an increase of the amplitude of the pulse while in the second phase the pulse decreases to zero. From the start of the first phase to the end of the second phase, as a result of energy transferred to the system the initial conditions in system change and the vibrating motion starts. At the moment when the free vibrations start, the third phase begins - just after the end of the pulse, local deformations in the vibrating system fade away. This phenomenon is visible between samples 1 and 4 in Fig. 1.

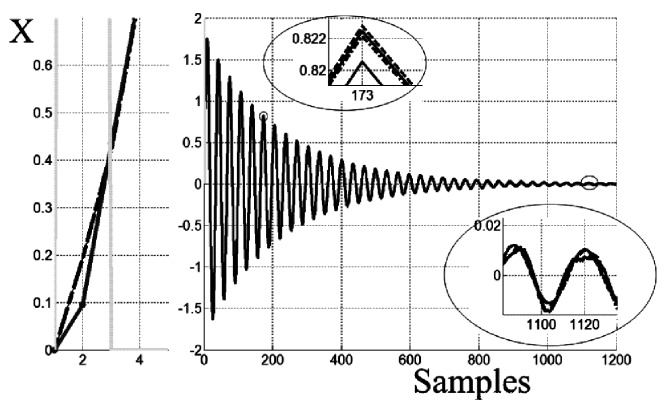

Fig. 1. The waveform representing vibrations of an RLC circuit ( $L=5 \mathrm{mH}, C=5 \mathrm{nF}$ ) for three different experiments. On the left, the first four recorded samples of the RLC circuit response are zoomed in, on the right the whole course of the system response is shown. The solid, the dashed, and the dashed-dotted line correspond to results of experiments $\# 1, \# 2$, and $\# 3$, respectively. The gray solid line represents mathematical model.

The phenomenon of the occurrence and disappearance of local deformations influences the waveform of the registered response of the real system. In connection with this, a question arises at which moment the waveform of the motion forced by the impulse ends and the free vibrations of the system start. The answer to this question is connected with approximation of the registered response $\boldsymbol{X}$ of the system, representing a series (vector) of measurements.

An approximation of the recorded waveform $\boldsymbol{X}$ is obtained in the MATLAB environment. On the basis of subsequent amplitudes of vibrations, two parameters, $b$ and $\eta$, are approximated with the help of the function fit with the parameter ' $\exp 1$ ', and the function $X_{1}=\eta \exp (-b t)$ is computed. The time vector $\boldsymbol{t}_{\boldsymbol{M}}$ corresponds to the sampling sequence and consequently its components assume values $t_{M, i}=0 \mathrm{~s}, 10^{-6} \mathrm{~s}, 2 \times 10^{-6} \mathrm{~s}$, ... where $t_{M, 1}$ is the instant of time when the impulse has its highest value.

By dividing the registered response $\boldsymbol{X}$ of the system by $X_{1}$, the parameter $c$ can be determined, e.g. with the help of function $f$ it with the parameter ' $\sin 1$ '.

All methods of approximation for numerous varied cases of $\boldsymbol{X}$ executed with the help of the function $f i t$ with the parameters ' $\sin 1$ ' or ' fourier 1 ' consistent with the expected mathematical model (4) fail (Table I), because what is dealt with here is a phase shift $\tilde{\phi}$ and the system responds with motion described by

$$
x(t)=\tilde{\eta} \exp (-\tilde{b} t) \sin (\tilde{c} t+\tilde{\phi}),
$$

where the tilde over the symbol of a quantity denotes it approximate value.

The algorithms approximating the response of the system to an impulse forcing require determination of a new 


\section{TABLE I}

Approximated parameters of the response depending on the starting point of the approximation.

\begin{tabular}{c|c|c}
\hline \hline parameter & \multicolumn{2}{|c}{ The starting point is } \\
& the first sample & the second sample \\
\hline$\tilde{b}$ & 4628.09 & 4628.09 \\
$\tilde{\eta}$ & 1.819073 & 1.810942 \\
$\tilde{c}$ & 192371.17 & 19284.41 \\
$\tilde{\phi}$ & -0.134892 & 0.054419
\end{tabular}

time vector $\boldsymbol{t}_{\boldsymbol{m}}$ for the values $x$ adopted in the model (5). The value of the new time vector component $t_{m, 1}$ is sought among those samples of the recorded response $\boldsymbol{X}$ for which the approximated angle $\tilde{\phi}$ changes from the negative to positive value. The search continues until the angle $\tilde{\phi}$ is close to zero with an assumed precision. The new time vector is created starting from $t_{\boldsymbol{m}, 1}$ with the step $10^{-6} \mathrm{~s}$ and for the time vector established this way, values $x$ according to model (5) are computed.

\section{Approximation of vibrations of an RLC circuit}

The goal of approximation of the response of a system with the help of the function in the form $X=\eta \mathrm{e}^{-b t} \sin (c t)$ is a most accurate possible representation of the relationship describing the response of a real system with one degree of freedom to a pulse forcing.

Approximation is carried out by way of comparison of:

- the vector $\boldsymbol{X}$ representing response of a system recorded at discrete instants of time (points of the time axis)

- with values $x$ determined in the model for the domain of time for which the angle $\tilde{\phi}$ is close to zero,

and then through computing the difference between corresponding components of $\boldsymbol{X}$ and values of $x$. During the calculation, the unknown parameters of the function $x$ that are searched for are $\eta, b$, and $c$, as well as $t_{M, 1}$ (the starting time of free vibrations).

The algorithms of approximation for determining of the hypothesis approximating the target function require repetitive processing of the whole practice set. The learning process for these algorithms [9] is divided into a number of repetitions, each of which includes a single processing of a practice example. To make it possible, the algorithm requires determination of the boundary values of the desired parameters. The values of parameters $\eta$ and $b$ presented in Table II are established as the boundary parameters of the practice set.

The fact that the determined dependence of the target function on the parameters is burdened with the errors resulting from the complexity of the function, imprecision of measurement, digitization of the signal, and differences
TABLE II

Two practice sets processed while determining the hypothesis approximating the target function.

\begin{tabular}{c|c|c|c|c|c|c}
\hline \hline & \multicolumn{3}{|c|}{ practice set \#1 } & \multicolumn{3}{c}{ practice set \#2 } \\
\cline { 2 - 7 } & start & step & end & start & step & end \\
\hline$\eta$ & 1.772624 & 0.001809 & 1.844976 & 1.799760 & 0.000452 & 1.817848 \\
$b$ & 4572.23 & 2.31 & 4664.59 & 4604.58 & 0.58 & 4627.66
\end{tabular}

between the model and the physical phenomenon must be taken into account. The total error of approximation can be minimized through selection of the algorithm presented below.

The algorithm of determining the desired parameters is realized the MATLAB environment according to the following scheme:

1. The values of the practice set - initial values of the parameters are determined.

2 . For the analysis, two subsequent parameters, $\eta$ and $b$,are selected. It is necessary to select these parameters because approximation is burdened with an error resulting from digitization of the signal it is not possible to find the actual maximum and minimum values among the recorded amplitudes; only the highest and the lowest ones can be found.

3. For the selected parameters, the function $X_{1}=$ $\eta \exp (-b t)$ is computed and the recorded response of the system is divided by $X_{1}$.

4. For the obtained function $X_{2}=\sin (c t)$, the parameter $c$ and the angle $\phi$ for two different points are determined with the help of the function $f i t\left(\mathrm{~d} t, y,{ }^{\prime} \sin 1^{\prime}\right)$. Between two samples from the recorded response of the system, the phase angle changes its sign from negative to positive value. The point for which the value of angle $\phi$ is close to zero with a certain accuracy is searched for using the search interval halving. In this way not only the parameter $c$ is calculated, but also $t_{\boldsymbol{m}, 1}$, i.e. the time at which one sample of free vibrations has been recorded.

5. For the RCL system selected for study, the vibrations fade out after 1200 registered samples. Due to the fact that small deflections are burdened with a high uncertainty of measurements (Fig. 1), the mean difference between the physical system $X_{i}$ and the model $x_{i}$ is computed for the first 1000 samples,

$\delta_{\mathrm{I}}=\frac{1}{n} \sum_{i=1}^{n}\left\|X_{i}|+| x_{i}\right\|$,

and saved in a file.

6. The process is reiterated starting from step 2 . 
The result of execution of this algorithm can be seen in Fig. 2 for three different experiments. The distribution of differences depending on the parameters $b$ and $\eta$ for the first and second practice set is presented in left and right column, respectively. The refinement of the practice set for the area in which the smallest differences between the model and the physical system were registered does not significantly reduce the calculated differences in any of the three presented experiments. A similar phenomenon can be observed while analyzing vibrations of other RLC circuits.

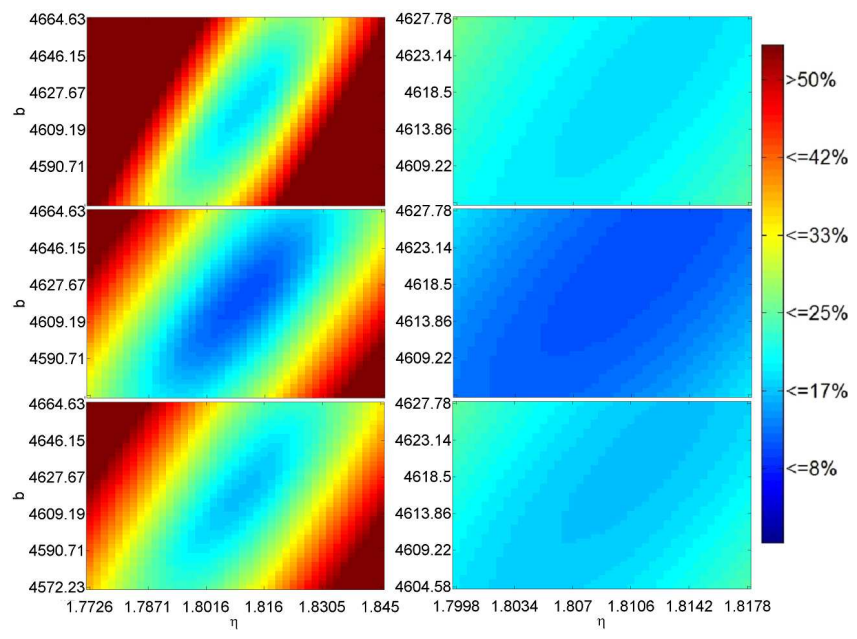

Fig. 2. The differences between approximated response of the RLC circuit and the registered one for the three experiments, calculated with the help of the formula (7), shown in left and right panels for the first and second practice set, respectively.

It is usually linear models that are studied in practice, since it is easy to analyze them; in this case we deal with damping that changes during vibrations. The response of the RLC circuit is not consistent with model (5), which probably follows from the very nature of physical systems and is not a result of forcing discordant with theoretical assumptions. It is also possible that through introduction of the measuring card and cables in the RLC circuit, its characteristics gets changed.

Starting from that moment, the parameters of the RLC circuit are no longer sought but what is searched for is the parameters that could substitute the actual response of the system with a linear model.

The non-linear nature of the phenomenon results in that determining of the parameters by way of searching for the minimum values in the tables presented in Fig. 2 does not bring about the expected results. It can be observed when the search criterion in step 5 of the algorithm is substituted with

$$
\delta_{\mathrm{II}}=\frac{1}{n} \sum_{i=1}^{n}\left(\left|X_{i}\right|-\left|x_{i}\right|\right) .
$$

One more criterion is also proposed to be used:

$$
\delta_{\mathrm{III}}=\frac{1}{n} \sum_{i=1}^{n}\left(X_{i}^{2}-x_{i}^{2}\right) .
$$

Registering the mean difference (8) and (9) between the model $x$ and the physical system $X$ as well as using white and gray color to mark the areas where the differences are greater or less than zero, respectively, it can be seen that the diagrams below differ in the inclination angle of the curve dividing the positive part from the negative one. Fig. 3 presents results of the first experiment only, since the other two lead to the similar picture.

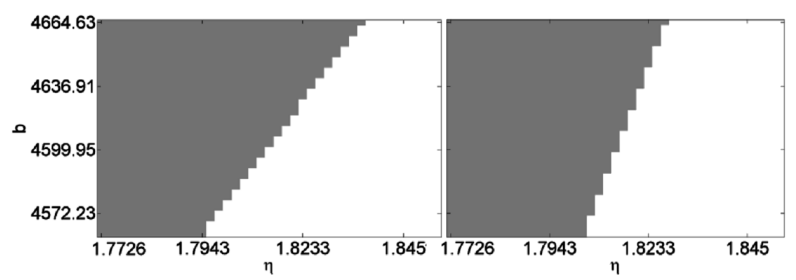

Fig. 3. The computed differences between the recorded response of the RLC circuit and the approximated one for the first experiment, computed with the use of (8) and (9), and shown in left and right panel, respectively.

By plotting straight line separating the negative part from the positive one and comparing the obtained results depending on whether the algorithm calculates the differences from the start or from the subsequent two samples

$$
\begin{aligned}
& \delta_{\mathrm{II}}=\frac{1}{n-n_{\text {start }}+1} \sum_{i=n_{\text {start }}}^{n}\left(\left|X_{i}\right|-\left|x_{i}\right|\right), \\
& \delta_{\text {III }}=\frac{1}{n-n_{\text {start }}+1} \sum_{i=n_{\text {start }}}^{n}\left(X_{i}^{2}-x_{i}^{2}\right),
\end{aligned}
$$

where $n_{\text {start }}=1,2$, or 3 , the effect of local deviations occurring in the RLC circuit on the approximation can be assessed.

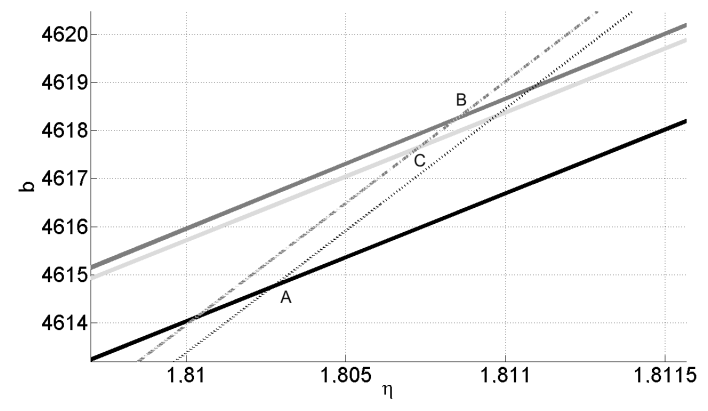

Fig. 4. Straight lines demarcating positive and negative differences computed with the use of $(8)$ — solid lines and (9) - dashed/dotted lines, with characteristic points A, B, and C marked. See text for explanation.

In Fig. 4 presenting such dividing lines for values $x$ and $x^{2}$, one can distinguish three characteristic points:

1. A is the crossing point of black lines, the solid and the dotted one. It is at this point that the change of 
negative values to positive ones occurs, both from the difference of $x$ and $X$, and the difference of $X^{2}$ and $x^{2}$ approximated from sample \#1.

2. B is the crossing point of dark gray lines, the solid and the dashed-dotted one. At this point, the change of negative values to positive ones occurs, both from the difference of $x$ and $X$, and the difference of $X^{2}$ and $x^{2}$ approximated from sample \#2.

3. $\mathrm{C}$ is the crossing point of light gray lines, the solid and dotted one. At this point the change from negative to positive values occurs, both from the difference of $x$ and $X$, and the difference of $X^{2}$ and $x^{2}$ approximated from sample $\# 3$.

The precise parameters obtained from the approximations for points $\mathrm{A}, \mathrm{B}$ and $\mathrm{C}$ are presented in Table III.

TABLE III

The approximated parameters of the response obtained from the approximation for the points $\mathrm{A}, \mathrm{B}$, and $\mathrm{C}$.

\begin{tabular}{|c|c|c|c|c|c|c|}
\hline \multirow[t]{2}{*}{ exp. } & \multirow[t]{2}{*}{ point } & \multicolumn{5}{|c|}{ parameters } \\
\hline & & $b$ & $\eta$ & $n_{\text {start }}$ & $t_{M}$ & $c$ \\
\hline \multirow{9}{*}{$\# 1$} & \multirow{3}{*}{ A } & \multirow{3}{*}{4614.76} & \multirow{3}{*}{1.810272} & 1 & 0.2781 & 192392.03 \\
\hline & & & & 2 & 0.2736 & 192394.63 \\
\hline & & & & 3 & 0.2742 & 192394.28 \\
\hline & \multirow{3}{*}{ B } & \multirow{3}{*}{4618.25} & \multirow{3}{*}{1.810849} & 1 & 0.2779 & 192392.14 \\
\hline & & & & 2 & 0.2735 & 192394.74 \\
\hline & & & & 3 & 0.2741 & 192394.38 \\
\hline & \multirow{3}{*}{$\mathrm{C}$} & \multirow{3}{*}{4617.97} & \multirow{3}{*}{1.810732} & 1 & 0.2780 & 192392.13 \\
\hline & & & & 2 & 0.2735 & 192394.73 \\
\hline & & & & 3 & 0.2741 & 192394.38 \\
\hline \multirow{9}{*}{$\# 2$} & \multirow{3}{*}{ A } & \multirow{3}{*}{4618.27} & \multirow{3}{*}{1.810167} & 1 & 0.2779 & 192392.14 \\
\hline & & & & 2 & 0.2735 & 192394.74 \\
\hline & & & & 3 & 0.2741 & 192394.38 \\
\hline & \multirow{3}{*}{ B } & \multirow{3}{*}{4621.30} & \multirow{3}{*}{1.810548} & 1 & 0.2778 & 192392.24 \\
\hline & & & & 2 & 0.2733 & 192394.84 \\
\hline & & & & 3 & 0.2740 & 192394.47 \\
\hline & \multirow{3}{*}{$\mathrm{C}$} & \multirow{3}{*}{4620.96} & \multirow{3}{*}{1.810635} & 1 & 0.2778 & 192392.23 \\
\hline & & & & 2 & 0.2733 & 192394.83 \\
\hline & & & & 3 & 0.2740 & 192394.46 \\
\hline \multirow{9}{*}{$\# 3$} & \multirow{3}{*}{ A } & \multirow{3}{*}{4616.45} & \multirow{3}{*}{1.810265} & 1 & 0.2780 & 192392.09 \\
\hline & & & & 2 & 0.2735 & 192394.69 \\
\hline & & & & 3 & 0.2741 & 192394.33 \\
\hline & \multirow{3}{*}{ B } & \multirow{3}{*}{4620.09} & \multirow{3}{*}{1.810870} & 1 & 0.2779 & 192392.20 \\
\hline & & & & 2 & 0.2734 & 192394.80 \\
\hline & & & & 3 & 0.2740 & 192394.44 \\
\hline & \multirow{3}{*}{$\mathrm{C}$} & & & 1 & 0.2779 & 192392.19 \\
\hline & & 4619.86 & 1.810776 & 2 & 0.2734 & 192394.79 \\
\hline & & & & 3 & 0.2740 & 192394.43 \\
\hline
\end{tabular}

The comparison performed for the results of each experiment separately shows that the differences between the parameters obtained at points $\mathrm{A}, \mathrm{B}$, and $\mathrm{C}$ are significant. The obtained results are also affected by un- certainty of measurements as well as intervals between the measurements, which is shown by the analysis of the values obtained for the particular points $\mathrm{A}, \mathrm{B}$, and $\mathrm{C}$ in all three experiments.

The most important thing, however, is that the selection of the substitute parameters between these points should be executed by applying the criterion of the least differences $\delta_{\mathrm{II}}$ and $\delta_{\mathrm{III}}$. Table IV shows the differences $\delta_{\text {II }}$ and $\delta_{\text {III }}$ for $t_{M}$ and $c$ approximated from sample \#2 for $n_{\text {rmstart }}=1$ and 2 for 1000 samples.

TABLE IV

Differences $\delta_{\text {II }}$ and $\delta_{\text {III }}$ for $t_{M}$ and $c$ approximated from the 2 nd measurement sample for the variable $n_{\text {start }}=1$ and 2, for 1000 samples corresponding to points A, B, and $\mathrm{C}$ (see Fig. 4).

\begin{tabular}{c|c|c|c|c|c}
\hline \hline exp. & \multirow{2}{*}{ point } & \multicolumn{2}{|c|}{$n_{\text {start }}=1$} & \multicolumn{2}{c}{$n_{\text {start }}=2$} \\
\cline { 3 - 6 } & & $\delta_{\mathrm{II}}$ & $\delta_{\mathrm{III}}$ & $\delta_{\mathrm{II}}$ & $\delta_{\mathrm{III}}$ \\
\hline \multirow{3}{*}{$\# 1$} & $\mathrm{~A}$ & $4.9982 \times 10^{-6}$ & $5.8863 \times 10^{-7}$ & $-9.2916 \times 10^{-5}$ & $-2.8797 \times 10^{-5}$ \\
\cline { 2 - 6 } & $\mathrm{B}$ & $1.0437 \times 10^{-4}$ & $2.0485 \times 10^{-5}$ & $6.5526 \times 10^{-6}$ & $-7.7032 \times 10^{-6}$ \\
\cline { 2 - 6 }$\# 2$ & $\mathrm{C}$ & $1.0605 \times 10^{-4}$ & $3.2650 \times 10^{-5}$ & $8.2239 \times 10^{-6}$ & $4.4728 \times 10^{-6}$ \\
\hline \multirow{3}{*}{$\# 2$} & $\mathrm{~A}$ & $4.8819 \times 10^{-6}$ & $-2.2980 \times 10^{-6}$ & $-9.3717 \times 10^{-5}$ & $-3.0765 \times 10^{-5}$ \\
\cline { 2 - 6 } & $\mathrm{B}$ & $1.0729 \times 10^{-4}$ & $3.9375 \times 10^{-5}$ & $8.7471 \times 10^{-6}$ & $1.0940 \times 10^{-5}$ \\
\hline & $\mathrm{C}$ & $7.8102 \times 10^{-5}$ & $9.3000 \times 10^{-6}$ & $-2.0468 \times 10^{-5}$ & $-1.9164 \times 10^{-5}$ \\
\hline \multirow{3}{*}{$\# 3$} & $\mathrm{~A}$ & $7.6931 \times 10^{-6}$ & $1.0924 \times 10^{-5}$ & $-9.0898 \times 10^{-5}$ & $-1.7529 \times 10^{-5}$ \\
\cline { 2 - 6 } & $\mathrm{B}$ & $1.1081 \times 10^{-4}$ & $3.2216 \times 10^{-5}$ & $1.2318 \times 10^{-5}$ & $3.7840 \times 10^{-6}$ \\
\hline & $\mathrm{C}$ & $1.1190 \times 10^{-4}$ & $4.1796 \times 10^{-5}$ & $1.3406 \times 10^{-5}$ & $1.3372 \times 10^{-5}$
\end{tabular}

In all the cases presented above, the parameters recorded for point $\mathrm{A}$ should be used; if the measurement taken from the first sample is taken into account in the considerations, it is the moment when the pulse reaches the system. If the first sample was neglected, determining the distribution of the values of stochastic pulses in a finite time would require taking into account of the parameters approximated for point B.

Unfortunately, for low intensities of strikes the differences $\delta_{\mathrm{II}}$ and $\delta_{\mathrm{III}}$ may turn out to be significant enough to result in computation leading to incorrect distributions of pulses.

\section{Conclusion}

The response of a physical system to forcing by a pulsed "hit" reveals features of a non-linear system. The standard search for a minimum among the differences between the values obtained in the model $x$ and the recorded response of the system $X$ is insufficient if the response of a physical system is to be replaced with a linear model. Moreover, it is necessary in each case to take into account the effect of deformations that occur while energy is injected to the system.

Approximation requires application of effective methods, and its results serve for an analysis aimed at selection of the parameters of the vibrating system so that the impact of the local deformations on the parameters obtained as a result of approximation is as small as possible.

\section{Acknowledgment}

This work has been financed by the Polish Ministry of Science and Higher Education, research project No. N N501 180636. 


\section{References}

[1] M. Jabłoński, A. Ozga, Acta Phys. Pol. A 118, 174 (2010).

[2] M. Jabłoński, A. Ozga, Mechanics 29, 163 (2010).

[3] M. Jabłoński, A. Ozga, T. Korbiel, P. Pawlik, Acta Phys. Pol. A 119, 997 (2011).

[4] M. Jabłoński, A. Ozga, Acta Phys. Pol. A 121, A174 (2012).

[5] Z. Osiński, Theory of Vibrations, PWN, Warszawa 1980, (in Polish).
[6] S. Kaliski, Vibrations and Waves in Solid Bodies, PWN, Warszawa 1966, (in Polish).

[7] M. Jabłoński, A. Ozga, Arch. Acoust. 34, 601 (2009).

[8] P. Cichosz, Machine Learning, WNT, Warszawa 2000, (in Polish).

[9] S. Piesiak, Identification of Mechanical Systems in the Domain of Non-linear and Degenerated Dynamic Models, Oficyna Politechniki Wrocławskiej, Wrocław 2003, (in Polish). 